\title{
Clonal dissemination of VanA-type glycopeptide-resistant Enterococcus faecalis between hospitals of two cities located $100 \mathrm{~km}$ apart
}

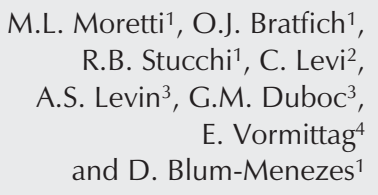

M.L. Moretti ${ }^{1}$, O.J. Bratfich ${ }^{1}$, R.B. Stucchi ${ }^{1}$, C. Levi², A.S. Levin ${ }^{3}$, G.M. Duboc ${ }^{3}$, E. Vormittag ${ }^{4}$ and D. Blum-Menezes ${ }^{1}$

\author{
'Laboratório de Epidemiologia Molecular e Moléstias Infecciosas, \\ Divisão de Moléstias Infecciosas, Faculdade de Ciências Médicas, \\ Universidade Estadual de Campinas, and ${ }^{2}$ Centro Médico de Campinas, \\ Campinas, SP, Brasil \\ ${ }^{3}$ Departamento de Moléstias Infecciosas, Faculdade de Medicina, \\ Universidade de São Paulo, São Paulo, SP, Brasil \\ ${ }^{4}$ Hospital Israelita Albert Einstein, São Paulo, SP, Brasil
}

\section{Correspondence}

M.L. Moretti

Divisão de Moléstias Infecciosas

FCM, UNICAMP

Rua Alexandre Fleming, 40

13081-970 Campinas, SP

Brasil

Fax: +55-19-3289-4107

E-mail: moretti@hc.unicamp.br

Publication supported by FAPESP.

Received November 18, 2003 Accepted June 14, 2004

\section{Abstract}

Nosocomial dissemination of glycopeptide-resistant enterococci represents a major problem in hospitals worldwide. In Brazil, the dissemination among hospitals in the city of São Paulo of polyclonal DNA profiles was previously described for vancomycin-resistant Enterococcus faecium. We describe here the dissemination of VanA phenotype E. faecalis between two hospitals located in different cities in the State of São Paulo. The index outbreak occurred in a tertiary care university hospital (HCUSP) in the city of São Paulo and three years later a cluster caused by the same strain was recognized in two patients hospitalized in a private tertiary care hospital (CMC) located $100 \mathrm{~km}$ away in the interior of the state. From May to July 1999, 10 strains of vancomycin-resistant $E$. faecalis were isolated from 10 patients hospitalized in the HCUSP. The DNA genotyping using pulsed-field gel electrophoresis (PFGE) showed that all isolates were originated from the same clone, suggesting nosocomial dissemination. From May to July 2002, three strains of vancomycin-resistant E. faecalis were isolated from two patients hospitalized in $\mathrm{CMC}$ and both patients were colonized by the vancomycin-resistant Enterococcus in skin lesions. All isolates from CMC and HCUSP were highly resistant to vancomycin and teicoplanin. The three strains from CMC had minimum inhibitory concentration $>256 \mu \mathrm{g} / \mathrm{ml}$ for vancomycin, and 64 (CMC 1 and CMC 2) and $96 \mu \mathrm{g} / \mathrm{ml}$ (CMC 3) for teicoplanin, characterizing a profile of VanA resistance to glycopeptides. All strains had the presence of the transposon $T n 1546$ detected by PCR and were closely related when typed by PFGE. The dissemination of the E. faecalis VanA phenotype among hospitals located in different cities is of great concern because E. faecalis commonly colonizes the gastrointestinal tract of patients and healthy persons for periods varying from weeks to years, which, together with the persistence of vancomycin-resistant Enterococcus in hospital rooms after standard cleaning procedures, increases the risk of the dissemination and reservoir of the bacteria.
Key words

- Vancomycin-resistant

Enterococcus

- Enterococcus faecalis

- Tn1546

- Nosocomial transmission

- São Paulo

........................ 
Nosocomial infections caused by glycopeptide-resistant Enterococcus spp are a growing problem in hospitals worldwide. Since the first cases of vancomycin-resistant Enterococcus (VRE) were described in 1988 $(1,2)$ an increasing number of cases have been reported in several hospitals in the world $(3,4)$, including Brazil $(5)$. The molecular epidemiology of VRE has shown that a single predominant strain was recovered in hospital settings right after the first identification of VRE $(6,7)$ and a polyclonal profile was reported in hospitals where VRE had been present for some time $(8,9)$. The VanA phenotype VRE strains are typically highly resistant to vancomycin and moderately to highly resistant to teicoplanin (10). Inducible resistance to high levels of vancomycin and teicoplanin is mediated by the transposon $T n 1546$ or closely related elements (11). In the present investigation, we studied the inter-hospital dissemination of VanA-type E. faecalis strains that occurred between two hospitals located in two different cities 100 $\mathrm{km}$ apart in the State of São Paulo, Brazil.

Hospitals settings. Centro Médico de Campinas (CMC) is a private tertiary care hospital located in Campinas, SP, and 100 $\mathrm{km}$ from the capital of the State of São Paulo. Hospital das Clínicas of the University of São Paulo (HCUSP) is a tertiary care teaching hospital associated with the University Medical School with approximately 2000 beds and is the main reference hospital in São Paulo, a city of approximately 11 million inhabitants.

E. faecalis vancomycin-resistant clinical isolates. From May to July 1999, 10 vancomycin-resistant strains of E. faecalis were isolated from 10 patients admitted to HCUSP. Vancomycin-resistant E. faecalis was isolated from urine $(\mathrm{N}=6)$, from a central venous catheter $(\mathrm{N}=1)$, from feces $(\mathrm{N}=1)$, and from bronchoalveolar lavage $(\mathrm{N}=1)$. The DNA genotyping by pulsed-field gel electrophoresis (PFGE) showed that all isolates originated from the same clone, sug- gesting nosocomial dissemination (12).

From May to July 2002, 3 strains of vancomycin-resistant $E$. faecalis were isolated from 2 patients hospitalized at CMC and both patients were colonized by VRE in skin lesions. All isolates were tested for susceptibility to vancomycin and teicoplanin using the Etest ${ }^{\circledR}$ and minimum inhibitory concentrations (MIC) were determined according to the NCMLS document M100S10(M7) 2000 (13).

Control strains of E. faecalis. During the period from 1999 to 2002, 60 strains of vancomycin-susceptible $E$. faecalis stored in $10 \%$ skim milk at $-20^{\circ} \mathrm{C}$ were included in the present study as control strains for DNA typing by PFGE. These strains were isolated from patients with bloodstream infections admitted to CMC.

Detection of transposon Tn 1546 . Tn 1546 was detectd by PCR based on the method of Martineau et al. (14). The isolates were cultured in BHI broth and $2 \mu \mathrm{l}$ of $0.5 \mathrm{McF}$ arland solution of the bacterial suspension was transferred to a PCR solution with a $50-\mu l$ final volume ( $2.5 \mathrm{mM} \mathrm{MgCl}_{2}, 0.1 \%$ Triton X-100, $2.5 \mathrm{mM}$ dNTP, $2.5 \mathrm{U}$ Taq polymerase, 50 pmol of each primer: 5'TTA TAA CCG TTC CCG CAG AC and 5'GAA ACC CAG ATT GA). PCR conditions were: $3 \mathrm{~min}$ at $92^{\circ} \mathrm{C}, 30$ cycles of denaturation at $92^{\circ} \mathrm{C}$ for 1 min, annealing temperature at $60^{\circ} \mathrm{C}$ and extension at $72^{\circ} \mathrm{C}$ for $1 \mathrm{~min}$, and a final extension at $72^{\circ} \mathrm{C}$ for $3 \mathrm{~min}$. PCR products were electrophoresed on $1.5 \%$ agarose gel. The primers were designed on the basis of the Tn 1546 sequence deposited in Genbank under accession number M97297.

Genomic DNA typing by PFGE. DNA extraction was performed as previously described (15). The genetic relatedness among the strains was interpreted by the method of Tenover et al. (12) and isolates with identical or related PFGE patterns were considered to be derived from a common clone. The genetic relationship among PFGE patterns was also analyzed by computer soft- 
ware after capturing the autoradiographic images with an IS-1000 digital imaging system (Bio-Capt MW, version 99; M\&S Instruments Trading Inc., Marne-la Valle, France). The dendrogram of the PFGE patterns was generated using the Dice coefficient (Biogene software; Vilbert-Loumart, Marne-la-Valle, France).

All isolates from CMC and HCUSP were highly resistant to vancomycin and teicoplanin. The three strains from CMC had MIC $>256 \mu \mathrm{g} / \mathrm{ml}$ for vancomycin, and 64 (CMC 1 and CMC 2) and $96 \mu \mathrm{g} / \mathrm{ml}$ (CMC 3) for teicoplanin, characterizing a profile of $\mathrm{VanA}$ resistance to glycopeptides.

The genomic DNA profiles obtained by PFGE revealed that all strains from HCUSP had the same profile, which was closely related (derived from the same clone, according to Tenover et al., 12) to that of the strains from CMC (Figure 1). Comparison of the E. faecalis strains from the two hospitals showed that the CMC strains were closely related to those from HCUSP, suggesting that they came from the same clone. The relationship among the E. faecalis strains shown in the dendrogram (Figure 2) demonstrated that the strains from the hospitals were $95 \%$ related. Among the E. faecalis control strains, we found one clone with three strains possibly related to the VanAresistant strains.

All vancomycin-resistant strains were submitted to PCR amplification of the 168bp fragment corresponding to Tn1546 (Figure 3), characterizing the VanA phenotype of the strains from both hospitals. The three vancomycin-susceptible isolates possibly related to the VRE clone had no amplification of the 168-bp fragment.

Since the identification of cases of VanAresistant Enterococci in Brazil $(16,17)$, subsequent cases and nosocomial dissemination of VRE were described in the city of São Paulo, especially related to the E. faecium VanA phenotype (5). Zanella et al. (18) described the characterization of a large group of VRE in Brazil during the investigation of a nosocomial outbreak in a general hospital in the city of São Paulo. However, interhospital dissemination of the E. faecalis VanA phenotype seemed to be less common than dissemination of the E. faecium VanA phenotype.

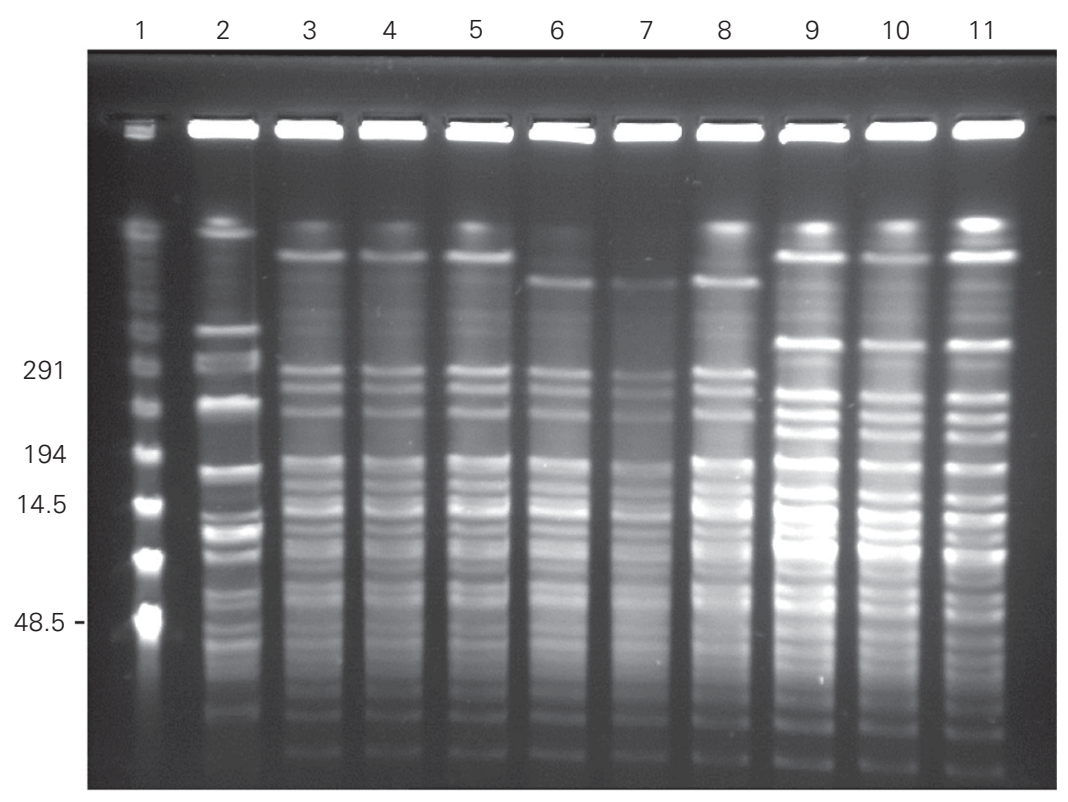

Figure 1. PFGE of Enterococcus faecalis. Lane 1, Molecular weight lambda ladder size marker (kb); lane 2, E. faecalis ATCC 29212; lanes 3 to 5, vancomycin-resistant E. faecalis isolated from Hospital das Clínicas, Universidade de São Paulo; lanes 6 to 8, vancomycinresistant $E$. faecalis from Centro Médico de Campinas; lanes 9 to 11, vancomycin-susceptible $E$. faecalis isolated from bloodstream infections at Universidade Estadual de Campinas (control strains).

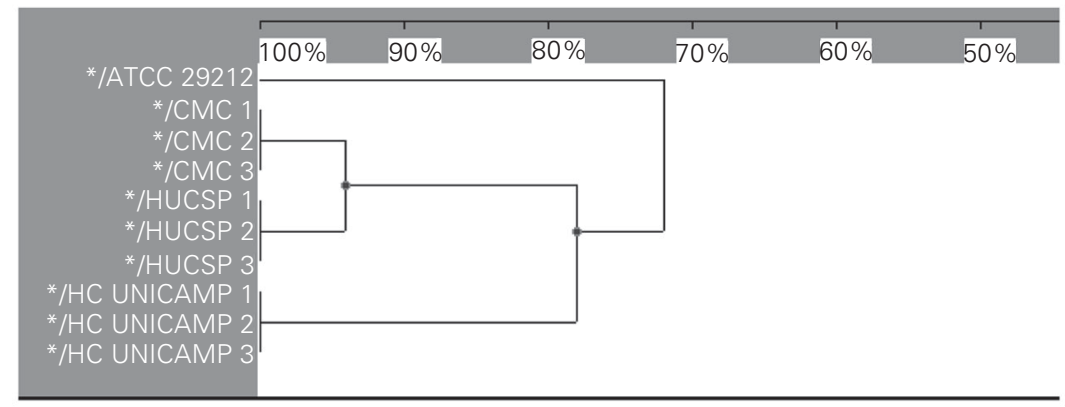

Figure 2. Dendrogram of Enterococcus faecalis VanA phenotype. The values were generated from the Dice coefficient showing the similarity of $E$. faecalis vancomycin-resistant strains from Centro Médico de Campinas (CMC), Hospital das Clínicas, Universidade de São Paulo (HCUSP) and vancomycin-susceptible E. faecalis from Universidade Estadual de Campinas (UNICAMP). 


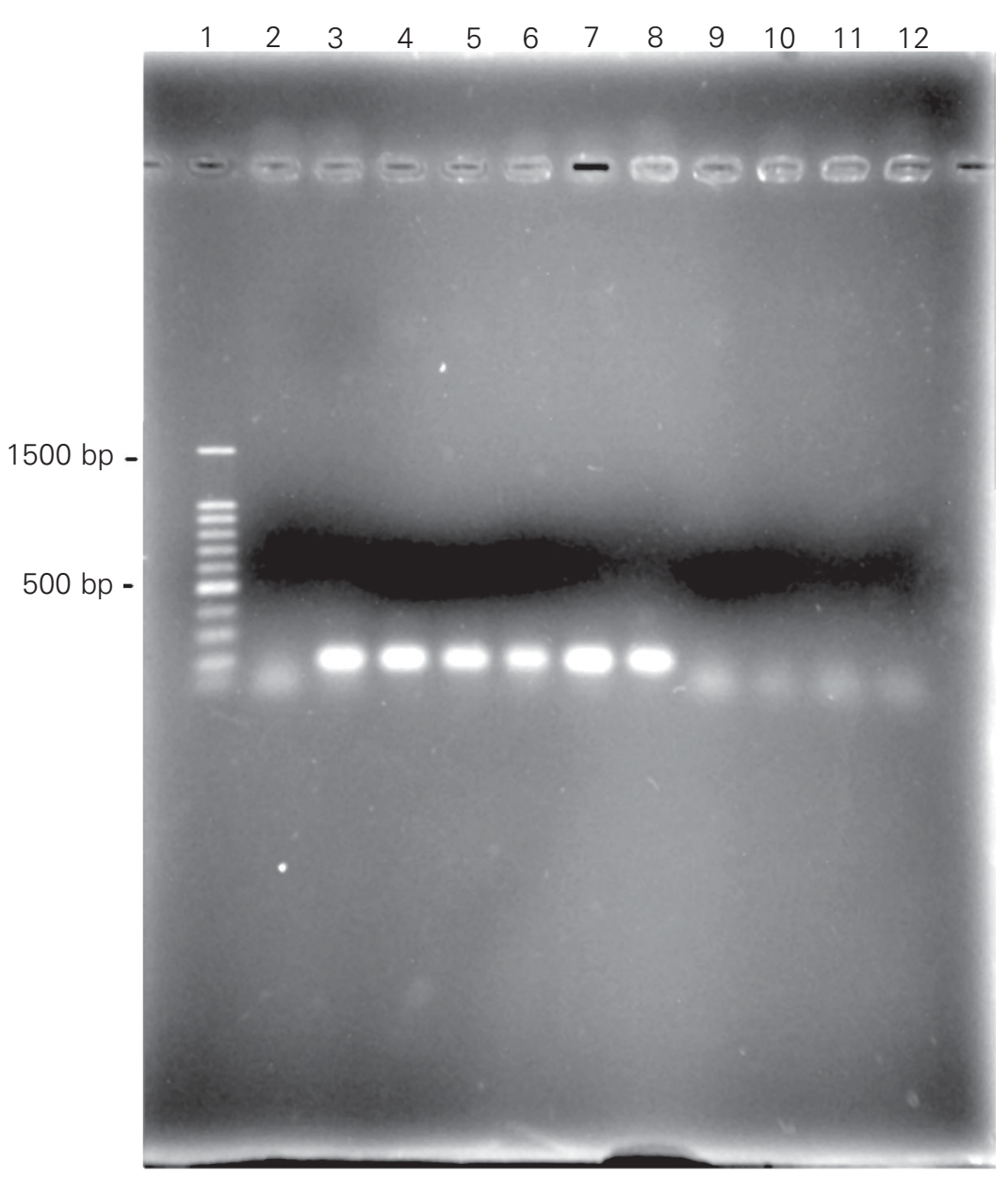

Figure 3. Demonstration of transposon Tn1546 in vancomycin-resistant isolates of Enterococcus faecalis by PCR. The 168-bp product corresponded to the amplification of Tn1546. Lane 1, Molecular weight in bp; lane 2, E. faecalis ATCC 29212; lanes 3 to 5, vancomycinresistant E. faecalis from Hospital das Clínicas, Universidade de São Paulo; lanes 6 to 8, E. faecalis vancomycin resistant from Centro Médico de Campinas; lanes 9 to 11, E. faecalis vancomycin-susceptible from Universidade Estadual de Campinas; lane 12, negative control.
The molecular typing study of the VRE isolated in 1999 from HCUSP showed intrahospital dissemination. The emergence of the two cases of VRE in the hospital in Campinas indicated a possible inter-hospital dissemination. DNA typing showed that the two clusters were closely related and strongly suggests an inter-hospital dissemination involving the spread of VRE in different cities in Brazil. Of note, the cluster in CMC occurred three years after the detection of the VRE cases in HCUSP.

The origin of VRE in CMC was unclear but appeared to have been imported from HCUSP. The strains were closely related genomically, suggesting intra-hospital dissemination. A previous study on intra-hospital VRE dissemination involved E. faecium strains from 6 medical centers in São Paulo, showing a polyclonal DNA profile and intra-hospital dissemination in the city of São Paulo (5). However, the dissemination of the E. faecalis VanA profile among hospitals involving different cities in Brazil has not been described previously. This event is of great concern because E. faecalis commonly colonizes the gastrointestinal tract of patients and healthy persons for periods varying from weeks to years (19); the persistence of VRE in hospital rooms after standard cleaning procedures increases the risk of the dissemination and reservoir of the bacteria (20).

\section{References}

1. Leclercq R, Derlot E, Durval J \& Courvalin P (1988). Plasmid-mediated resistance to vancomycin and teicoplanin in Enterococcus faecium. New England Journal of Medicine, 319: 157-161.

2. Uttley AHC, Collins CH, Naidoo J \& George RC (1988). Vancomycinresistant enterococci. Lancet, 1: 57-58.

3. Murray BE (2000). Vancomycin-resistant enterococcal infections. New England Journal of Medicine, 342: 710-721.

4. Jones RN, Sader HS, Erwin ME \& Anderson SC (1995). Emerging multiply resistant enterococci among clinical isolates. I. Prevalence data from 97 medical center surveillance study in the United States. Enterococcus Study Group. Diagnostic Microbiology and Infectious Disease, 21: 85-93.

5. Cereda RF, Gales AC, Silbert S, Jones RN \& Sader HS (2002). Molecular typing and antimicrobial susceptibility of vancomycin resistant Enterococcus faecium in Brazil. Infection Control and Hospital Epidemiology, 23: 19-22.

6. Biavasco F, Miele A, Vignaroli C, Manso E, Lupid R \& Varaldo PE (1996). Genotypic characterization of a nosocomial outbreak of VanA Enterococcus faecalis. Microbial Drug Resistance, 2: 231-237.

7. Boyce JM, Opal SM, Chow JW, Zervos MJ, Potter-Bynow G, Sherman CB, Romulo RL, Fortna $S$ \& Medeiros AA (1994). Outbreak of multidrug-resistant Enterococcus faecium with transferable VanB class vancomycin resistance. Journal of Clinical Microbiology, 32: 1148-1153.

8. Boylen JF, Soumakis SA, Rendo A, Herrington JA, Gianarkis DG, Thurberg BE \& Painter BF (1993). Epidemiologic analysis and genotypic characterization of a nosocomial outbreak of vancomycinresistant enterococci. Journal of Clinical Microbiology, 31: 1280- 
1285.

9. Morris JG, Shay DK, Hebden JN, McCarter Jr RJ, Perdue BE, Jarvis W, Johnson JA, Dowling TC, Polish LB \& Schwalbe RS (1995). Enterococci resistant to multiple antimicrobial agents, including vancomycin. Annals of Internal Medicine, 123: 250-259.

10. Arthur M, Reynolds P \& Courvalin P (1996). Glycopeptide resistance in enterococci. Trends in Microbiology, 4: 401-407.

11. Murray B (1998). Diversity among multi-drug resistant Enterococci. Emerging Infectious Diseases, 4: 37-47.

12. Tenover FC, Arbeit RD, Goering RV, Mickelsen PA, Murray B, Persing DH \& Swaminathan B (1995). Interpreting chromosomal DNA restriction patterns produced by pulsed field gel electrophoresis: criteria for bacterial strain typing. Journal of Clinical Microbiology, 33: 2233-2239

13. National Committee for Medical Laboratory Standards (2000). Methods for dilution antimicrobial susceptibility test for bacteria that grow aerobically. MIC testing, approved standard M100-S10(M7). 5th edn. Wayne, PA, USA.

14. Martineau F, Picard FJ, Roy PH, Ouellette M \& Bergeron MG (1996). Species-specific and ubiquitous DNA-based assays for rapid identification of Staphylococcus epidermidis. Journal of Clinical Microbiology, 34: 2888-2893.

15. Van den Braack N, van Belkun A, van Keulen M, Vligenharat J, Verbrugh HA \& Faudtz HP (1998). Molecular characterization of vancomycin resistant enterococci from hospitalized patients and poultry products in The Netherlands. Journal of Clinical Microbiology, 36: 1927-1932.

16. Dalla Costa LM, Souza DC, Martins LT, Zanella RC, Brandileone MC, Bokermann S, Sader HS \& Souza HA (1998). Vancomycin-resistant Enterococcus faecium: first case in Brazil. Brazilian Journal of Infectious Diseases, 2: 160-163.

17. Zanella RC, Valdetaro F, Lovgren M, Tyrrel GJ, Bokermann S, Almeida SC, Vieira VS \& Brandileone MC (1999). First confirmed case of vancomycin-resistant Enterococcus faecium with VanA phenotype from Brazil: isolation from a meningitis case in São Paulo. Microbial Drug Resistance, 5: 159-162.

18. Zanella RC, Brandileone MCC, Bokermann S, Almeida SC, Valdetaro $F$, Vitório F, Moreira Mde F, Villins M, Salomão R \& Pignatari AC (2003). Phenotypic and genotypic characterization of VanA Enterococcus isolated during the first nosocomial outbreak in Brazil. Microbial Drug Resistance, 9: 283-291.

19. Henning KJ, Delencastre H, Eagan J, Boone N, Brown A, Chung M, Wollner N \& Armstrong D (1996). Vancomycin-resistant Enterococcus faecium on a pediatric oncology ward: duration of stool shedding and incidence of clinical infection. Pediatric Infectious Disease Journal, 15: 848-854.

20. Byers KE, Durbin LJ, Simonton MB, Anglim AM, Adal KA \& Farr BM (1998). Disinfection of hospital rooms contaminated with vancomycin-resistant Enterococcus faecium. Infection Control and Hospital Epidemiology, 19: 261-264. 